\title{
Different Regimes for Water Wave Turbulence
}

\author{
P. Cobelli, ${ }^{*}$ A. Przadka, and P. Petitjeans \\ Physique et Mécanique des Milieux Hétérogènes PMMH, UMR CNRS 7636-ESPCI-UPMC Univ. Paris 6-UPD Univ. Paris 7 , \\ Paris, France \\ G. Lagubeau and V. Pagneux \\ Laboratoire d'Acoustique de l'Université du Maine, UMR CNRS 6613, Avenue Olivier Messiaen, 72085 Le Mans, France
}

A. Maurel

Institut Langevin, LOA, UMR CNRS 7587, ESPCI, UPD Univ. Paris 7, 10 rue Vauquelin, 75231 Paris Cedex 05, France

(Received 20 July 2011; published 18 November 2011)

\begin{abstract}
We present an experimental study on gravity capillary wave turbulence in water. By using space-time resolved Fourier transform profilometry, the behavior of the wave energy density $\left|\eta_{\mathbf{k}, \omega}\right|^{2}$ in the $3 \mathrm{D}(\mathbf{k}, \omega)$ space is inspected for various forcing frequency bandwidths and forcing amplitudes. Depending on the bandwidth, the gravity spectral slope is found to be either forcing dependent, as classically observed in laboratory experiments, or forcing independent. In the latter case, the wave spectrum is consistent with the Zakharov-Filonenko cascade predicted within wave turbulence theory.
\end{abstract}

DOI: 10.1103/PhysRevLett.107.214503

PACS numbers: 47.27. $-\mathrm{i}, 47.35 . \mathrm{Bb}$

Nonlinear wave interactions are ubiquitous in all domains of physics [1]. Among these, weak nonlinear random waves are especially interesting since they are able to experience an energy cascade through different scales. This has been described in the framework of wave turbulence theory, which, in contrast to classical hydrodynamic turbulence, possesses analytical predictions for the power law scaling of the energy density spectrum [2-4]. Note also the work of [5] on the interaction of bulk hydrodynamic turbulence and free surface. There is still debate about whether the physics of ocean waves is described well by the wave turbulence theory (WT) even though this appears to fit the spectra measured for ocean waves in many cases $[6,7]$. To gain deeper insight into the concordance between the theory and measurements, well-controlled laboratory experiments have been developed in recent years [8-13]. These experiments have raised the question of whether or not the conditions for the applicability of WT theory can be in place in such finite systems. Indeed, this theory assumes weak nonlinearities, low attenuation, and small finite size effects, and meeting these requirements is particularly difficult in a laboratory tank [13-16].

In the present Letter, we present space-time resolved experimental results of different regimes of wave turbulence.

The joint space-time power spectrum,

$$
\left|\eta_{\mathbf{k}, \omega}\right|^{2} \equiv \int d \mathbf{r} d t\left\langle\eta\left(\mathbf{r}^{\prime}, t^{\prime}\right) \eta\left(\mathbf{r}+\mathbf{r}^{\prime}, t+t^{\prime}\right)\right\rangle e^{i(\omega t+\mathbf{k} \cdot \mathbf{r})}
$$

shows how the nonlinear interactions spread the wave energy in the 3D $(\mathbf{k}, \omega)$ space. This quantity fills the gap between the $k$-space point of view of WT theory and the $\omega$ space which is usually more accessible in experiments (even though the link between the wave action variables used in the theory and the observable measurable quantities is not obvious [17]). Note that several attempts have previously been made to directly compute the wave number spectra $[8,11,18]$. The joint space-time power spectrum allows us to test the validity of the premise of WT theory that assumes the concentration of the wave energy on the renormalized dispersion relation (which remains close to the linear dispersion relation) and it allows us to evaluate the isotropy of the energy distribution. We obtained different turbulence regimes by varying the forcing frequency range $\left[0, \omega_{m}\right]$. In one case, the wave field is shown to be composed only of resonant free modes whose energy is concentrated on the dispersion relation and the spectra appear to be in good agreement with the ZakharovFilonenko (ZF) spectra [19]. In the other case, the wave field is shared between the free modes and the nonresonant slave modes [17] (also called bound waves [20]) and our observations are similar to those of most of the laboratory experiments, with the existence of an inertial range that is dependent on the forcing amplitude [10,12,13].

In our experiments, water waves are generated by two piston-type wave makers ( $20 \mathrm{~cm}$ large and $1 \mathrm{~cm}$ immersed) in a $(177 \times 61) \mathrm{cm}^{2}$ tank filled with water with depth at rest $h_{0}=5 \mathrm{~cm}$. The wave maker motions are controlled by a random signal within a broadband frequency range $\left[0, \omega_{m}\right]$ with maximum amplitude $A$. We use excitations with typically $A$ between 1 and $30 \mathrm{~mm}$ and $\omega_{m}=25.1 \mathrm{~s}^{-1}$ (4 $\mathrm{Hz}$, the experiment is hereafter referred to as experiments I) and $\omega_{m}=9.44 \mathrm{~s}^{-1}(1.5 \mathrm{~Hz}$, hereafter experiments II). Note that the transition between gravity and capillary waves corresponds to a wave number $k_{c} \equiv$ $\sqrt{\rho g / \gamma}=369 \mathrm{~m}^{-1}\left(\omega_{c}=85 \mathrm{~s}^{-1}\right)$. 
Waves were measured by an optical method (Fourier transform profilometry) that we recently adapted for free surface characterization [21,22] and previously used in the context of wave turbulence in an elastic plate [23] (see also [24]). A full space-time characterization of the velocity field is performed. In the direct space, our spatial resolutions in both directions are the size of the projected pixel ( 0.2 or $0.4 \mathrm{~mm}$ in our experiments) and the temporal resolution is $1 / F$, with $F$ the acquisition frequency of the high-speed camera $(F=250 \mathrm{~Hz}$ in experiments I and $40 \mathrm{~Hz}$ in experiments II). The inspected field is $45 \times$ $45 \mathrm{~cm}^{2}$, with $968^{2}$ pixels and the measurements are performed over 6000 successive time steps. The resulting resolutions in the spectral space are $\Delta k=4.44 \pi \mathrm{m}^{-1}$ and $\Delta \omega=0.42$ or $2.61 \mathrm{~s}^{-1}$ (with 600 successive time steps in order to permit 10 averages). Finally, the accessible range of wave numbers is limited by the phase demodulation by $k / 2 \pi<1 / \lambda_{F}=200 \mathrm{~m}^{-1}$, with $\lambda_{F}$ the wavelength of the projected fringes. The sensitivity is improved by treating the phase shifts between two successive images (rather than the phase shift with a reference to unperturbed free surface), resulting in the measurement of the velocity of the surface elevation $\dot{\eta}(\mathbf{r}, t)$. Typical fields and the corresponding renormalized probability density functions are shown in Fig. 1 for both experiments I and II. In order to evaluate if it is close to a Gaussian distribution [25], we calculate the skewness $S \equiv\left\langle\dot{\eta}(\mathbf{r})^{3}\right\rangle / \sigma^{3}$ and the kurtosis $K \equiv\left\langle\dot{\eta}(\mathbf{r})^{4}\right\rangle / \sigma^{4}$ [with $\sigma \equiv \sqrt{\left\langle\dot{\eta}(\mathbf{r})^{2}\right\rangle}$ the variance]. In experiments I, the field is typically non-Gaussian with $S=0.15$ and $K=3.54$. On the other hand, in experiments II, it is fairly Gaussian with $S=0.023$ and $K=2.95$. This makes a priori experiments II a better candidate to satisfy the assumption of WT theory, but the full space-time characterization of the fields will give us deeper insight into the differences between the two experiments.

We first report the results obtained for experiments I, where the forcing is between 0 and $4 \mathrm{~Hz}$. The space-time power spectra of the velocity $\left|\dot{\eta}_{\mathbf{k}, \omega}\right|^{2}$ (and $\left|\dot{\eta}_{\mathbf{k}, \omega}\right|^{2}=$ $\left.\left|\eta_{\mathbf{k}, \omega}\right|^{2} / \omega^{2}\right)$ are computed by a multidimensional Fourier transform [Eq. (1)]. The isotropy of $\left|\dot{\eta}_{\mathbf{k}, \omega}\right|^{2}$ in the $\left(k_{x}, k_{y}\right)$ space is illustrated in inset (ii) of Fig. 2 (and it has been checked that the isotropy is preserved at all frequencies. By averaging over the direction $\theta_{k}$ of $\mathbf{k}$, one obtains the wave energy spectrum $\left|\eta_{k, \omega}\right|^{2}=\int d \theta_{k} k\left|\eta_{\mathbf{k}, \omega}\right|^{2}$ (main plot of Fig. 2), where the wave energy appears to be mainly concentrated on the linear dispersion relation $\omega(k)$,

$$
\omega^{2}(k)=g k \tanh \left(k h_{0}\right)\left(1+\gamma k^{2} / \rho g\right) .
$$

Nevertheless, as shown in inset (i) of Fig. 2, the wave energy is shared between the resonant part of the wave field on the linear dispersion relation $\omega(k)$ and a nonresonant part composed of slave modes (with $\omega \rightarrow N \omega, k \rightarrow$ $N k$ ) [17]. These latter modes, also called bound waves,
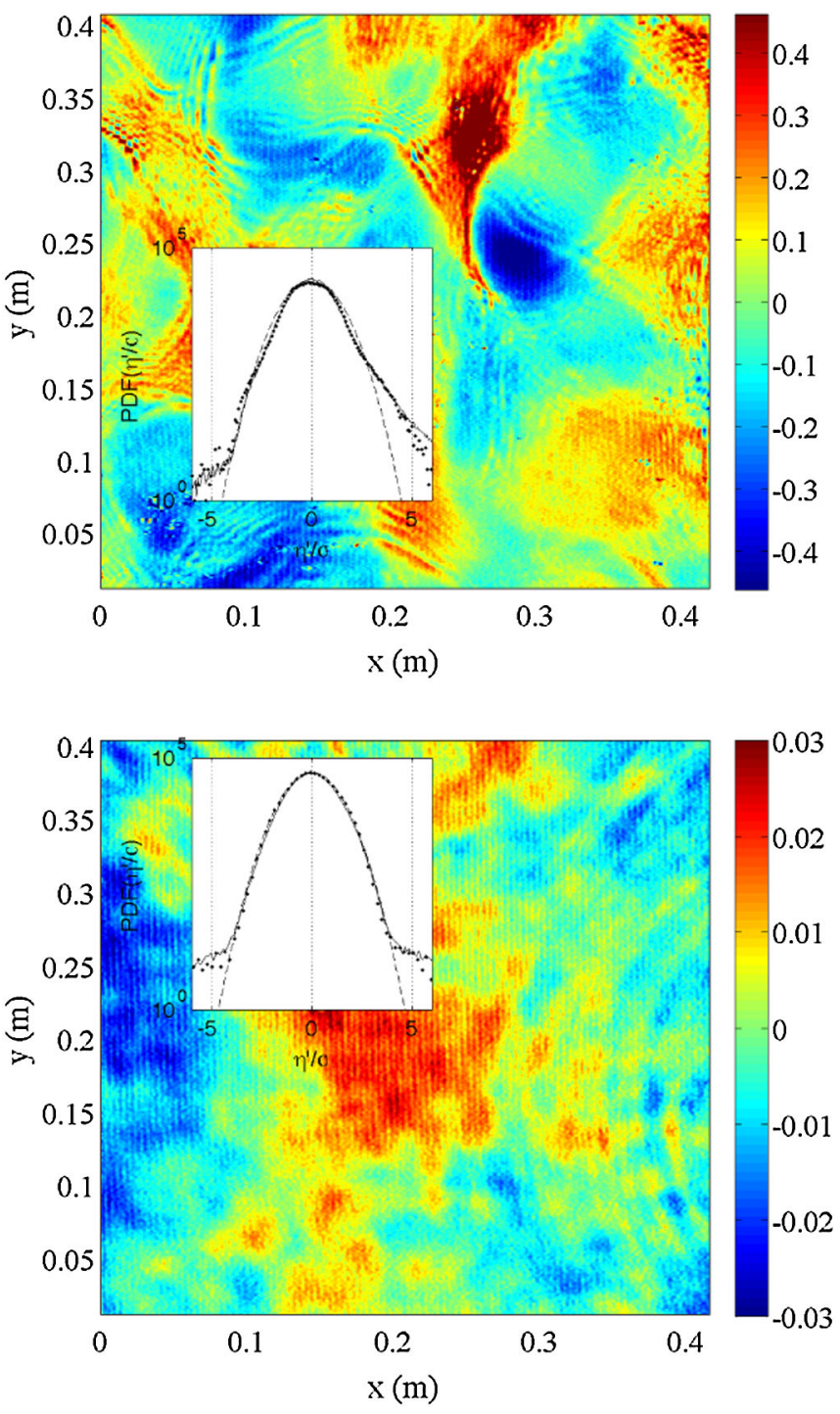

FIG. 1 (color online). Typical instantaneous velocity fields $\dot{\eta}(\mathbf{r}, t)$ (color scale is in $\mathrm{m} / \mathrm{s})$. Top: Experiments I $(A=$ $22 \mathrm{~mm})$, the variance $\sigma \equiv \sqrt{\left\langle\dot{\eta}(\mathbf{r})^{2}\right\rangle}=0.16 \mathrm{~m} / \mathrm{s}$, skewness $S \equiv$ $\left\langle\dot{\eta}(\mathbf{r})^{3}\right\rangle / \sigma^{3}=0.15$ and kurtosis $K \equiv\left\langle\dot{\eta}(\mathbf{r})^{4}\right\rangle / \sigma^{4}=3.54$. Bottom: Experiments II $(A=28 \mathrm{~mm}), \sigma=0.012 \mathrm{~m} / \mathrm{s}, S=$ $0.023, K=2.95$. The insets show the probability density functions of the normalized wave velocity $\dot{\eta}(\mathbf{r}) / \sigma$ : circles for the presented field, solid line when averaged over six fields at different times, and dotted line the Gaussian fit with mean zero and unit variance.

have already been observed numerically [16] and experimentally $[11,20]$.

The $(k, \omega)$ power spectra give the usual energy density spectra $\left|\eta_{\omega}\right|^{2}=\int d k\left|\dot{\eta}_{\mathbf{k}, \omega}\right|^{2} / \omega^{2}$ and $\left|\dot{\eta}_{k}\right|^{2}=$ $\int d \omega\left|\dot{\eta}_{\mathbf{k}, \omega}\right|^{2}$. WT theory for water waves predicts spectra for gravity waves,

$$
\left|\eta_{\omega}\right|^{2} \propto P^{1 / 3} \omega^{-4}, \quad\left|\eta_{k}\right|^{2} \propto P^{1 / 3} k^{-5 / 2},
$$

and for capillary waves, 


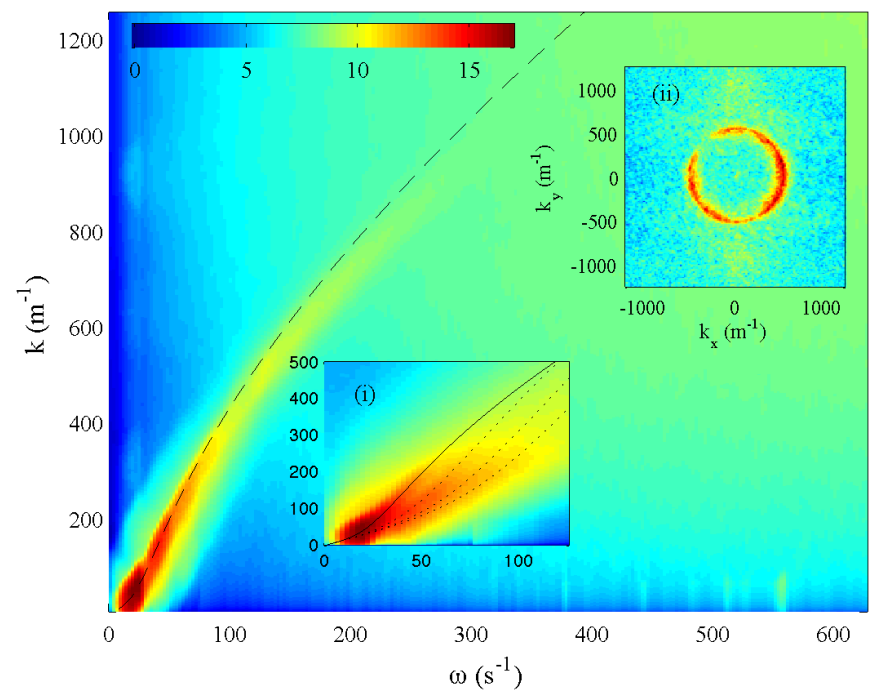

FIG. 2 (color online). Experiments I: Typical spectrum $\left|\eta_{k, \omega}\right|^{2}$ at low forcing $(A=3 \mathrm{~mm})$. Inset (i) shows a zoom of $\left|\eta_{k, \omega}\right|^{2}$ in the gravity wave regime at high forcing $(A=22 \mathrm{~mm})$. The solid line shows the linear dispersion relation $\omega(k)$ and the dotted lines the dispersion relation of the slave modes $N=2,3,4$. Inset (ii) illustrates the isotropy in the $\left(k_{x}, k_{y}\right)$ space by plotting $\left|\eta_{\mathbf{k}, \omega}\right|^{2}$ for $\omega=138 \mathrm{~s}^{-1}(A=3 \mathrm{~mm})$.

$$
\left|\eta_{\omega}\right|^{2} \propto P^{1 / 2} \omega^{-17 / 6}, \quad\left|\eta_{k}\right|^{2} \propto P^{1 / 2} k^{-15 / 4},
$$

where $P$ is the injected energy. Figure 3 displays the energy density spectra for experiments I. The inertial ranges for

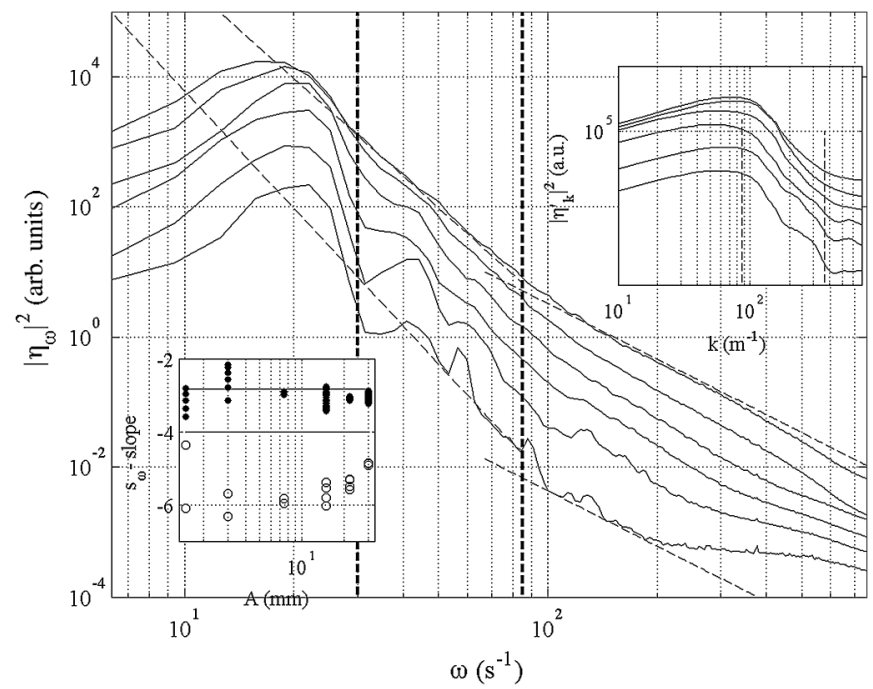

FIG. 3. Spectra $\left|\eta_{\omega}\right|^{2}$ in experiments I for $A=1.5,3,7.5,15$, 22 , and $30 \mathrm{~mm}$. Vertical dotted lines at $30 \mathrm{~s}^{-1}$ and at $\omega_{c}=$ $85 \mathrm{~s}^{-1}$ delimitate the inertial range of the gravity regime. Top inset: Corresponding spectra $\left|\dot{\eta}_{k}\right|^{2}$. Bottom inset: Spectral exponent as a function of $A$; open circles in the gravity regime and plain circles in the capillary regime (multiple symbols correspond to multiple fits in a moving window within the inertial range). capillary waves $\left(k>k_{c}\right)$ are forcing independent, with a spectral slope close to the $-17 / 6$ value predicted by WT [Eq. (4)]. For gravity waves $\left(k<k_{c}\right)$, the spectral slope is forcing dependent, with values from -6 to -5 when increasing the forcing amplitude. Our results are consistent with most of the results of laboratory experiments: indeed, similar tendency, with increasing spectral slope values when increasing the forcing amplitude, has been observed recently by several authors $[10,12,13]$.

As suggested in [16], it is attractive to make the low frequency forcing bandwidth as narrow as possible to try (i) to enlarge the inertial range in the gravity regime and (ii) to reduce the strong nonlinearities produced near the injection scale. Therefore, we reduce the frequency bandwidth in the series of experiments II with the maximum frequency $\omega_{m}=9.4 \mathrm{~s}^{-1}$.

Figures 4 and 5 show the same quantities as for experiments I (Figs. 2 and 3). Clear differences between the two wave systems appear: the most significant is the existence of a forcing independent inertial range with scaling laws in agreement with the ZF predictions $\left|\eta_{\omega}\right|^{2} \propto$ $\omega^{-4}$. The spectra in the $k$ space are shown in the inset of Fig. 5, where the ZF prediction $\left|\dot{\eta}_{k}\right|^{2} \propto k^{-3 / 2}$ is displayed for comparison.

Another clear different feature is the absence of nonlinear branches in Fig. 4, where nonlinear shifts with respect to the linear dispersion relation are not measurable. That means that the wave fields are only composed of resonant harmonics that cascade through smaller scales remaining on the dispersion relation manifold.

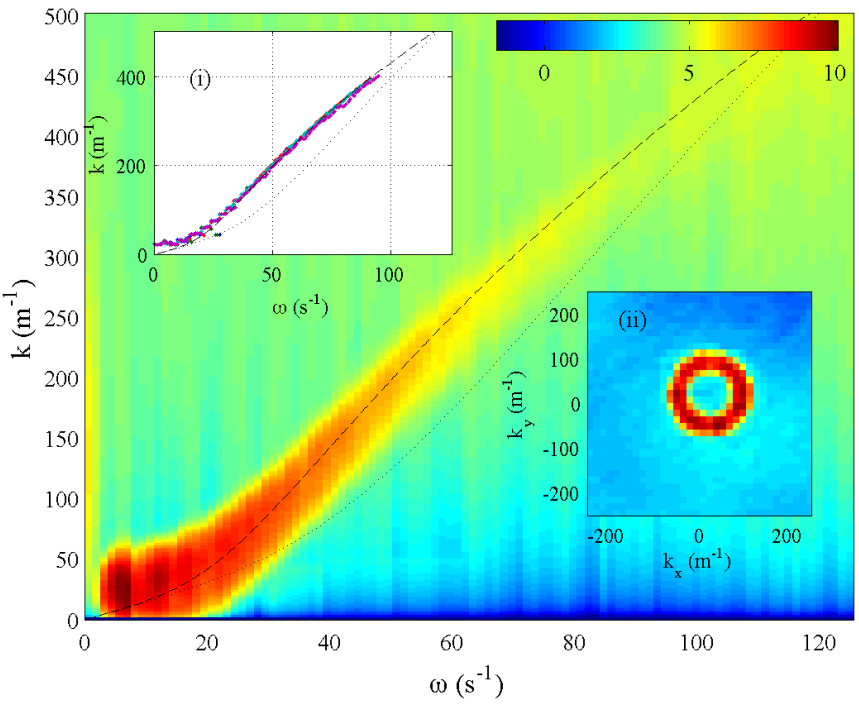

FIG. 4 (color online). Experiments II: Typical spectrum $\left|\eta_{k, \omega}\right|^{2}$ at forcing amplitude $A=20 \mathrm{~mm}$. Inset (i) shows the collected dispersion relations for $A=1,6,12,20$, and $28 \mathrm{~mm}$. The dashed line shows the linear dispersion relation and the dotted line the first nonlinear mode. Inset (ii) illustrates the isotropy in the $\left(k_{x}, k_{y}\right)$ space by plotting $\left|\eta_{\mathbf{k}, \omega}\right|^{2}$ for $\omega=$ $27.64 \mathrm{~s}^{-1}$. 


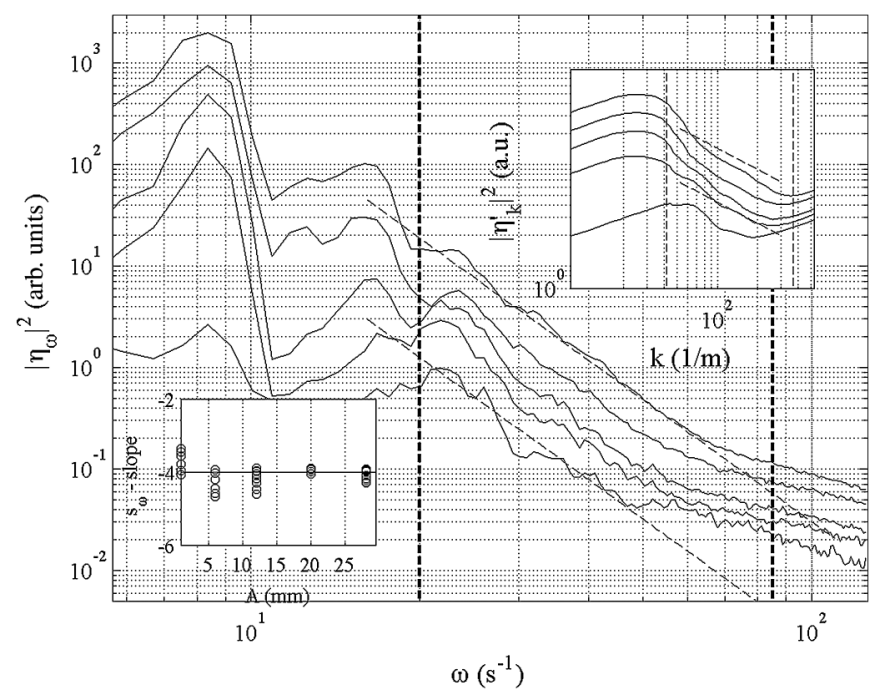

FIG. 5. Experiments II: Spectra $\left|\eta_{\omega}\right|^{2}$. Same representation as in Fig. 3 (only the gravity regime is accessible in these experiments).

WT theory [Eqs. (3) and (4)] predicts power laws in $\omega$ and $k$ as previously discussed and also power laws in the injected power $P$. A direct measurement of $P$ can be done in decaying turbulence, as in [12]. Otherwise, an estimate is given by the scaling $P \propto \omega_{m}^{2} A^{2}$ obtained in [10]. Another estimate is the maximum of the energy spectrum $\left|\eta_{k}\right|_{\max }^{2}$ that is shown to be proportional to $\omega_{m}^{2} A^{2}$ in the inset of Fig. 6; therefore, we use this latter estimate for $P$. Figure 6 shows typical values of the spectral amplitudes in the inertial ranges $\left|\eta_{k}\right|_{\mathrm{IR}}^{2}$ as a function of $P$ for both experiments I and II. In experiments I, we observe the same behavior as in previous experiments of the literature [10], with a linear power law with respect to $P$, both in the gravity and in the capillarity regimes. More interestingly, in experiments II, the power law with $P^{1 / 3}$ is a better fit than the power law with $P^{1}$, in agreement with WT theory.

Our work exemplifies the complexity of determining the parameters relevant to describe the weak or strong nonlinearities of turbulent wave fields in finite systems as used in laboratory experiments. Evidently, the loss of sensitivity to $k$-space discreteness and the associated resonance broadening necessary for the approximate four free waves interaction are expected to occur for large wave intensities. On the other hand, at large wave intensities, the appearance of bound modes may produce deviations with respect to the WT theory. In our experiments, we have changed the wave intensity for two forcing frequency ranges, and we observed two different regimes of wave turbulence. In experiments I, with broader forcing bandwidth, the presence of bound waves at large intensities indeed produce a deviation with respect to WT theory, but decreasing the wave intensity to eliminate the bound waves does not permit us to approach the WT prediction. In experiments II, with narrow forcing bandwidth, no bound

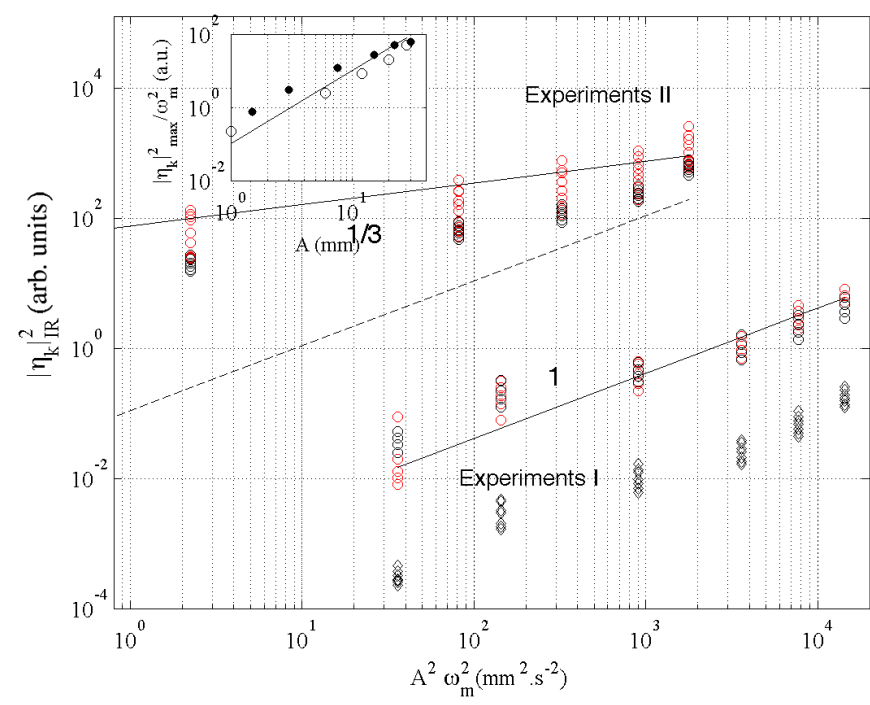

FIG. 6 (color online). Scaling laws of $\left|\eta_{k}\right|^{2}$ in the inertial range as a function of the forcing amplitudes $\omega_{m}^{2} A^{2} \propto P$. Black symbols come from $\left|\dot{\eta}_{k}\right|^{2}$ and gray (red) symbols are deduced from $\left|\dot{\eta}_{\omega}\right|^{2} d \omega / d k$ with $d \omega / d k$ determined from the linear dispersion relation. In experiments I, results are shown for both the gravity (circles) and capillary (diamond) regimes, and in experiments II, for the gravity regime. Solid and dotted lines have slope 1 and $1 / 3$. The inset shows $\left|\eta_{k}\right|_{\max }^{2} / \omega_{m}^{2}$ as a function of $A$ (solid line has a slope 2), suggesting that the injected power is proportional to $A^{2}$ in our experiments.

waves are generated for the intensities accessible experimentally, and the wave system appears to be in good agreement with WT theory, but we have not been able to further increase the wave intensity to see the appearance of bound waves. The difference between these two regimes remains an open question. Further works are needed to better understand the transition between both regimes, for instance, by changing continuously the forcing frequency bandwidth.

The authors thank the Agence Nationale de la Recherche for its funding under Grant No. Tourbillonde ANR-08BLAN-0108-02.

*Present address: Departamento de Fisica, FCEN, UBA and IFIBA, CONICET, Ciudad Universitaria, 1428 Buenos Aires, Argentina.

[1] G. B. Whitham, Linear and Nonlinear Waves (John Wiley \& Sons, New York, 1974).

[2] V.E. Zakharov, V.S. Lvov, and G. Falkovich, Kolmogorov Spectra of Turbulence (Springer-Verlag, Berlin, 1992).

[3] S. Nazarenko, Wave Turbulence, Lecture Notes in Physics Vol. 825 (Springer, New York, 2011).

[4] A. C. Newell and B. Rumpf, Annu. Rev. Fluid Mech. 43, 59 (2011).

[5] R. Savelsberg and W. van de Water, Phys. Rev. Lett. 100, 034501 (2008). 
[6] V. E. Zakharov, Direct and Inverse Cascades in the WindDriven Sea, http://math.arizona.edu/ zakharov/1Articles/ Cascades.pdf.

[7] L. Cavaleri et al. (WISE Group), Progr. Oceanogr. 75, 603 (2007).

[8] W. B. Wright, R. Budakian, and S. J. Putterman, Phys. Rev. Lett. 76, 4528 (1996).

[9] G. V. Kolmakov et al., Phys. Rev. Lett. 93, 074501 (2004).

[10] E. Falcon, C. Laroche, and S. Fauve, Phys. Rev. Lett. 98, 094503 (2007); E. Falcon, Discrete Contin. Dyn. Syst., Ser. B 13, 819 (2010).

[11] E. Herbert, N. Mordant, and E. Falcon, Phys. Rev. Lett. 105, 144502 (2010).

[12] P. Denissenko, S. Lukaschuk, and S. Nazarenko, Phys. Rev. Lett. 99, 014501 (2007).

[13] S. Lukaschuk, S. Nazarenko, S. McLelland, and P. Denissenko, Phys. Rev. Lett. 103, 044501 (2009); S. Nazarenko, S. Lukaschuk, S. McLelland, and P. Denissenko, J. Fluid Mech. 642, 395 (2009).

[14] E. Kartashova, Nonlinear Resonance Analysis (Cambridge University Press, Cambridge, England, 2010).

[15] E. Kartashova, S. Nazarenko, and O. Rudenko, Phys. Rev. E 78, 016304 (2008); V. S. Lvov and S. Nazarenko, Phys. Rev. E 82, 056322 (2010).
[16] Y. V. Lvov, S. Nazarenko, and B. Pokorni, Physica (Amsterdam) 218D, 24 (2006).

[17] V.E. Zakharov, Phys. Scr. T142, 014052 (2010).

[18] P. A Hwang et al., J. Phys. Oceanogr. 30, 2753 (2000); D. W. Wang and P. A. Hwang, J. Atmos. Ocean. Technol. 21, 1936 (2004); M. V. Kosnik and V. A. Dulov, Meas. Sci. Technol. 22, 015504 (2011).

[19] V.E. Zakharov and N. N. Filonenko, Sov. Phys. Dokl. 11, 881 (1967); J. Appl. Mech. Tech. Phys. 8, 37 (1971).

[20] W. J Plant, J. Geophys. Res. 109, C10002 (2004); H.E. Krogstad and K. Trulsen, Ocean Dynam. 60, 973 (2010).

[21] A. Maurel, P. Cobelli, V. Pagneux, and P. Petitjeans, Appl. Opt. 48, 380 (2009); P. Cobelli, A. Maurel, V. Pagneux, and P. Petitjeans, Exp. Fluids 46, 1037 (2009).

[22] Recent improvements of the technique for the signal processing and the choice of the painting particles can be found in G. Lagubeau et al. (to be published) and A. Przadka et al. (to be published).

[23] P. Cobelli et al., Phys. Rev. Lett. 103, 204301 (2009).

[24] P. Cobelli, V. Pagneux, A. Maurel, and P. Petitjeans, Europhys. Lett. 88, 20006 (2009); J. Fluid Mech. 666, 445 (2011).

[25] Y. Choi et al., Phys. Lett. A 339, 361 (2005). 